\title{
Local Recovery and Failure Masking for Stencil-based Applications at Extreme Scales
}

\author{
Marc Gamell ${ }^{\dagger}$, Keita Teranishi ${ }^{\ddagger}$, Michael A. Heroux $x^{\ddagger}$, Jackson Mayo ${ }^{\ddagger}$, Hemanth Kolla ${ }^{\ddagger}$, \\ Jacqueline Chen ${ }^{\ddagger}$ and Manish Parashar ${ }^{\dagger}$ \\ ${ }^{\dagger}$ Rutgers Discovery Informatics Institute, Rutgers University, Piscataway, NJ, USA \\ $\{$ mgamell,parashar\}@cac.rutgers.edu \\ ${ }^{\ddagger}$ Sandia National Laboratories, Livermore, CA, Albuquerque, NM, USA, \\ \{knteran, maherou, jmayo, hnkolla, jhchen\}@sandia.gov
}

\begin{abstract}
Application resilience is a key challenge that has to be addressed to realize the exascale vision. Online recovery, even when it involves all processes, can dramatically reduce the overhead of failures as compared to the more traditional approach where the job is terminated and restarted from the last checkpoint. In this paper we explore how local recovery can be used for certain classes of applications to further reduce overheads due to resilience. Specifically we develop programming support and scalable runtime mechanisms to enable online and transparent local recovery for stencil-based parallel applications on current leadership class systems. We also show how multiple independent failures can be masked to effectively reduce the impact on the total time to solution. We integrate these mechanisms with the S3D combustion simulation, and experimentally demonstrate (using the Titan Cray-XK7 system at ORNL) the ability to tolerate high failure rates (i.e., node failures every 5 seconds) with low overhead while sustaining performance, at scales up to 262144 cores.
\end{abstract}

\section{Categories and Subject Descriptors}

1 [System Software]: Support for fault-tolerance and resilience; 2 [Performance]: Resilience, Workload characterization and benchmarking

\section{Keywords}

Fault-Tolerance, Performance Modeling, Scalable Methods

\section{INTRODUCTION}

The increasing demands of science and engineering applications continue to push the limits of current extremescale systems. As a result, the HPC community is working towards achieving exascale $\left(10^{18}\right.$ FLOPS) by the end the

ACM acknowledges that this contribution was authored or co-authored by an employee, or contractor of the national government. As such, the Government retains a nonexclusive, royalty-free right to publish or reproduce this article, or to allow others to do so, for Government purposes only. Permission to make digital or hard copies for personal or classroom use is granted. Copies must bear this notice and the full citation on the first page. Copyrights for components of this work owned by others than ACM must be honored. To copy otherwise, distribute, republish, or post, requires prior specific permission and/or a fee. Request permissions from permissions@acm.org.

SC'15, November 15-20, 2015, Austin, TX, USA

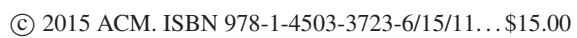

DOI: http://dx.doi.org/10.1145/2807591.2807672 decade $[1,13]$ or early next decade. One of the key anticipated challenges at exascale will be the reliability of the system, primarily due to the very large number of cores and components $[4,26,34]$. The mean time between failures (MTBF) for current petascale systems is measured in days (e.g., production runs on ORNL's Titan Cray showed 9 node failures/day, as shown in [15]), but it is estimated that the MTBF for an exascale system would be measured in minutes [13]. An important class of failures that must be addressed is process and node failures, including correlation effects. These failures are often recovered by terminating the job and restarting it from the last checkpoint found in stable storage. While coordinated, stable-storage-based global checkpoint/restart $(\mathrm{C} / \mathrm{R})$ is currently the most widely accepted technique for addressing processor failures, it is unclear whether this approach will scale to exascale since the time to checkpoint will often be longer than the expected MTBF, and researchers continue to actively address this issue. For example, runtimes that aim to offer an abstraction of a fault-free system to the application have been developed (e.g. MPICH-V [7]). However, as suggested by recent studies [19], the abstraction of a failure-free machine will not be sustainable at extreme scales, and application-aware resilience techniques will likely be required at exascale.

While programing models such as task-DAG can include resilience features, SPMD and message passing are not designed to handle process failures by default. In previous studies, [15] we have shown how online (i.e., without disrupting the job) global recovery (i.e., involving all the cores in the system in the recovery process) can be used in conjunction with application-guided checkpointing to support high failure rates (i.e., every 47 seconds) for message passing applications using the Fenix framework. In that approach, every failure triggered a global recovery, which required all survivor processes to recover the MPI environment. Then, all surviving processes, along with the newly spawned ones, had to rollback to the last commonly available checkpoint. The advantage of global recovery is that it can be done in a semi-transparent way: the application does not necessarily have to be aware of the failure. However, due to the intrinsic global nature of the recovery algorithms, global recovery presents scalability challenges.

In this paper we present the design, prototype implementation, and evaluation of local recovery approaches for certain classes of applications in FenixLR. Specifically we study the feasibility of local recovery for stencil-based paral- 
lel applications, which represent a significant set of physical simulations, and develop programming support and scalable runtime mechanisms, to enable online and transparent local recovery on current leadership class systems. In addition to its inherent scalability, local recovery provides several benefits. For example, the environment does not need to be recovered globally after a failure, and only the newly spawned processes have to rollback to the last checkpoint.

The key idea underlying the local recovery approach has been presented by [16]. Stencil-based parallel applications, such as simulations that solve partial differential equations (PDEs) using finite-difference methods, typically consist of a number of iterations (timesteps) with each iteration consisting of two key steps, computation on local data to advance the simulation, and communication with the immediate neighbors. This communication pattern implies that, upon failure, by allowing the rest of the domain to continue the simulation, only the immediate neighbors will be immediately affected by that failure. When a failure occurs, we can substitute the failed process with a spare one, rollback to the last saved state for the failed process (i.e., the last checkpoint), and resume computation for that process. In this paper we show how the effect of the failure will slowly propagate through the machine. If subsequent failures occurs at a distant node before the original failure delay has spread to that node, we demonstrate that the delay of the second failure will be masked with the delay of the first one. In general, we show that the overhead of several separate failures on the total execution time can appear to be as the overhead of a single failure (failure masking).

We have implemented these solutions in a new framework, FenixLR, and deployed them on the Titan Cray-XK7 production system (world's second fastest machine as of November 2014) at ORNL. FenixLR shares the same programming interface as Fenix [15], but due to the fact that recovering locally needs very different requirements than recovering globally, the rest of its architecture and implementation has been re-designed. We decided to implement FenixLR as a standalone runtime whose only dependencies are a $\mathrm{C}++$ compiler and the Cray uGNI library (i.e. no MPI runtime needed). Since it has been built from scratch, this has been a nontrivial task. The motivation behind this decision is that fault tolerant versions of MPI, such as ULFM [5,6], do not serve our requirements. Specifically, they are not capable to deliver local recovery constructs. We present an experimental evaluation of the effectiveness and scalability of local recovery in FenixLR using the S3D [11] stencil-based combustion application. S3D is a highly parallel method-of-lines solver for PDEs and is used to perform first-principles-based direct numerical simulations of turbulent combustion. It employs high order explicit finite difference numerical schemes for spatial and temporal derivatives, and realistic physics for thermodynamics, molecular transport, and chemical kinetics. S3D demonstrates good scalability up to nearly $200 \mathrm{~K}$ cores, and has been highlighted by [2] as one of five promising applications on the path to exascale.

Our results demonstrate FenixLR's ability to tolerate highfrequency dynamically injected node failures while maintaining sustained performance of S3D on scales up to 262144 cores. Our evaluation also explores extreme execution scenarios that may exist at exascale, where node failures occur with high frequency (i.e., as often as every 5 seconds). For example, when injecting node failures every 30 seconds, per- formance is sustained with $13.75 \%$ overhead when compared with a failure-free and checkpoint-free execution. Finally, we show that the programming overhead of using FenixLR is low, requiring less than 35 new, changed, or rearranged lines of code in S3D.

Key contributions of this work include: (1) A study of the applicability of local recovery approaches to stencil-based parallel applications, including a model to understand and estimate the propagation of recovery delay due to failures; (2) design and implementation of FenixLR runtime, and its deployment on the Titan Cray XK7 production system at ORNL; and, (3) an experimental evaluation of local recovery algorithms in FenixLR on Titan using the S3D combustion application demonstrating its ability to support sustained performance and scalability in spite of high frequency real node failures.

\section{BACKGROUND AND RELATED WORK}

Process and node failures and their characteristics have been well documented in $[34,37]$. Checkpoint and restart $(\mathrm{C} / \mathrm{R})[20-22]$ is the most widely used technique for implementing resilience for HPC systems. In this model, the application state is periodically saved (e.g., using BLCR [18] or application-level $\mathrm{C} / \mathrm{R}$ ) so that, upon failure, global rollback can be used to restart the application from the last globally committed checkpoint. This process is independent of the number of nodes affected by the failure, i.e., if a node or process failure occurs, all processes are typically forced to rollback to the previous strongly consistent checkpoint. In contrast, using local recovery in FenixLR, only failed processes need to rollback to the previous checkpoint.

Checkpoint coordination. Global consistency of locally-created checkpoints [20] is typically ensured using coordination protocols. Examples of coordination protocols include full coordination [14], non-blocking coordination $[9,10]$ or blocking coordination [12]. The main advantage of coordinated protocols is that they are application-agnostic and create globally consistent checkpoints. The major drawback, however, is the overhead due to process synchronization. Uncoordinated protocols [8] do not required synchronization during checkpoint creation, thus reducing overheads and allowing application imbalance. However, during recovery, a consistent global state has to be found by examining the checkpoints. As these protocols cannot guarantee checkpoint global consistency on recovery, all processes may end up rolling back to the beginning of the execution, i.e., the domino effect. Uncoordinated checkpointing protocols can leverage message logging to avoid the domino effect for piecewise deterministic applications [14], at the expense of logging all the application messages. For senddeterministic applications, only a subset of all the messages need to be logged $[17,31]$. Building on these ideas, the research presented in [17] proposes a technique for recovering only a subset of processes on failure, while avoiding the domino effect. Furthermore, by letting the application indicate when checkpoints are created, implicitly coordinated protocols presented in [15] enable the advantages of coordinated protocols (i.e., guaranteed checkpoint consistency) to be combined with those of uncoordinated protocols (i.e., local checkpointing without synchronization).

Our approach in FenixLR uses such implicit coordination, and, in order to enable local recovery, it logs a small set of 


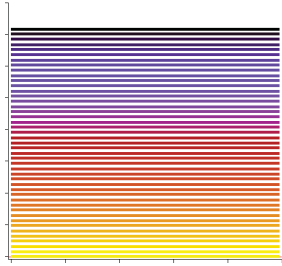

(a) No failures

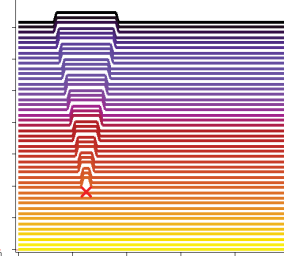

(b) One failure

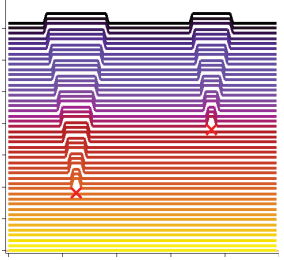

(c) Two failures

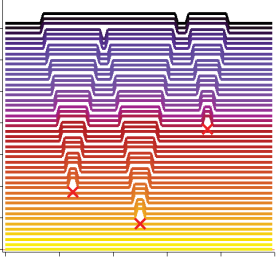

(d) Three failures

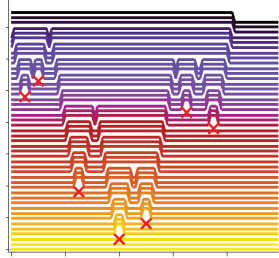

(e) Seven failures

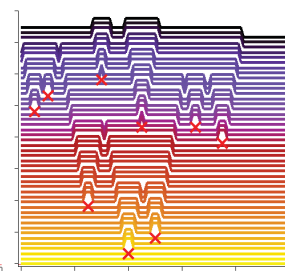

(f) Nine failures

Figure 1: Behavior of local recovery for a stencil-based 1-D partial differential equation (PDE) solver. X axis represents process number (or rank) and Y axis indicates wallclock time. Each line in a figure represents a timestep [16].

messages. The total size of these logged messages, for the target applications, is negligible when comparing with the size of the checkpoints. Note that using protocols such as those presented in [17] with our target applications (i.e., iterative applications with a stencil-based communication pattern), and assuming that the uncoordinated checkpoints are consistently created (i.e., the best case scenario), a process failure would require all processes of the system to rollback to the last checkpoint because orphan and rolled back message dependencies would extend across all of the mesh. This is not the case with our protocol.

Checkpoint storage. Typically, checkpoints are saved to a centralized parallel file system [20] but may also be stored in local memory [30], in both local and peer-memory [38], in non-volatile memory [25], in node-local storage (such as SSD) [3, 28], or at different storage layers [27]. They may be compressed [23], aggregated [29], or both [24]. In order to enable local recovery, FenixLR only needs to store checkpoints at a peer node. Other strategies, such as storing checkpoints in the parallel file system or compressing them would add performance overheads that makes them prohibitive for FenixLR, despite their advantages.

Combining optimized checkpointing with global recovery. Systems such as Fenix [15], LFLR [36] and FMI [32] show how advanced in-memory diskless checkpointing can be used in conjunction with global recovery to enable execution in a failure-prone scenario.

\section{LOCAL RECOVERY FOR STENCIL- BASED SCIENTIFIC APPLICATIONS}

This section briefly discusses the local recovery approach and our underlying reasoning for exploring this technique for stencil-based applications. Recovering from failures in a local manner implies that (1) only the re-spawned processes have to rollback to the last checkpoint and (2) only the processes that communicate with the failed ones will notice the failure and might be involved in the recovery process. These requirements are in contrast with global recovery, in which all the processes are involved in the recovery and rollback to the last consistent checkpoint. Global recovery can be costly and presents scalability challenges, and, in many situations, may be unnecessary. Note also that local recovery is by definition an online recovery technique, i.e. the job does not have to be disrupted.

In this section, we describe how the communication and computation patterns of stencil-based applications naturally fit to the local recovery description. Then, we address the practical merits and implementation challenges of the local recovery approaches for this class of applications. The details of our approach are also discussed in our previous work [16].

\subsection{Stencil-based Scientific Applications}

Stencil-based computation iteratively updates every mesh point of a discretized application domain defined by the stencil operator. In the parallel implementation for distributed memory systems, the application domain is typically partitioned into subdomains to assign the data and work into individual processes.

During the program execution, each process performs two key tasks at every timestep: (1) computation on its local data to advance the simulation, and (2) communication with its immediate neighbors based on the specific stencil used. In SPMD model, each process typically maintains a "ghost region" corresponding to the width of the stencil used around its local subdomain, and populates this region from its neighbors in a "ghost region exchange" communication step.

\subsection{Local Recovery, challenges and benefits}

The observation in the previous section indicates that process failures in stencil-based applications affect the computation of immediate neighbor subdomains of lost ones. This allows a natural adaptation of our local recovery approach, leaving the re-spawned process to (1) redo the local computation using the data at the previous timestep restored from the checkpoint, and (2) reestablish communication with the neighbors for the ghost region exchange to proceed the timestepping.

Consistency. Despite the relative simplicity of local recovery for target stencil-based applications, the challenge arises to guarantee consistency in message passing programming model as neighbor processes must communicate to make a progress. Our approach leverages ghost region exchange to implement a domain specific message logging, which keep the outgoing messages that have been transferred since the last checkpoint. In 5-point 2D stencil case, for example, four messages, are logged at each timestep. The overhead to maintain the log is negligible compared to checkpointing the local subdomains.

Delay Propagation. Process failures only triggers the neighbor processes to wait for the local recovery of the respawned processes, and the rest of the processes continue timestepping. Therefore, the delay from the recovery does not affect the rest of the processes immediately. In the next timestep, the second neighbors of the lost processes are delayed due to the delay of the first neighbors in the last timestep. As a result, the delay is propagated in diffu- 

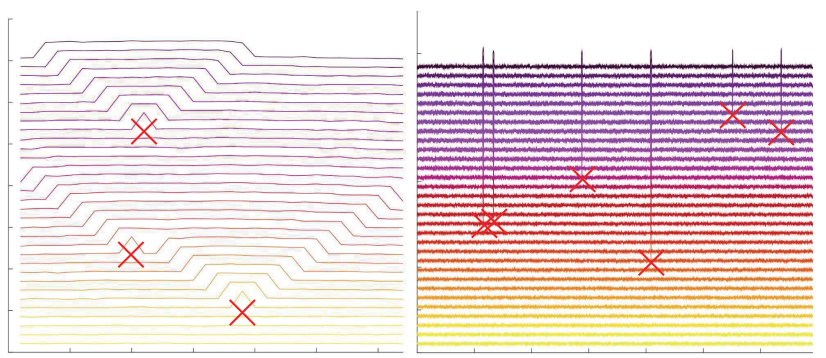

Figure 2: Results for delay propagation obtained from simulations based on the model for 32 cores (left) and 13824 cores (right). Each horizontal line indicates the same timestep across the nodes. ' $\mathrm{X}$ ' indicates the point of failure.

sive manner, showing wave-like shape in Figure 1(b) in 1D stencil case; this behavior also applies to higher dimensions. Failure masking. When using a large number of processes, it is possible that another failure occurs on distant processes where the delay from the first failure has not yet reached. In this case, the recovery delay of the second failure will begin propagating from the remote location, as see in Figure 1(c). At some point in space and time, the delay of both failures will merge. Consequently, the total delay will be the maximum of both delays. We call this effect failure masking, and an example can be seen in Figure 1(d). This situation is beneficial at large scales, because the impact of several failures on end-to-end execution time will be comparable to that of a single failure. Note that this effect can also happen with multiple failures, as seen in Figures 1(d) and 1(e). Comparing these four figures, we see that the total overhead is the same. This effect becomes more plausible with larger machines and lower MTBFs, which is an ideal property for good scalability.

There may be cases, however, where failures occur after the delay of previous failures have already reached the failed node. An example can be seen in Figure 1(f), in which the total execution time is comparable to that of recovering from two failures sequentially. The likelihood of this situation is dependent on the communication pattern of the application and the checkpointing approach used.

\section{MODELING DELAY PROPAGATION}

Section 3 covered the theoretical benefits of local recovery. This section goes one step further and tries to understand how these benefits can be mathematically modeled. Specifically, we construct a recurrence relation to simulate the execution pattern of a parallel stencil code and use it to quantify the impact of local recovery. Before moving on to the real evaluation in Section 6, this section will show how the presented equation can effectively model the behavior presented in Section 3.

\subsection{Modeling the delay for a 1-D stencil code}

As discussed in Section 3, in typical parallel stencil-based applications, every process communicates with its neighbors to exchange boundary data in each iteration. Based on this observation, we use the following recurrence relation

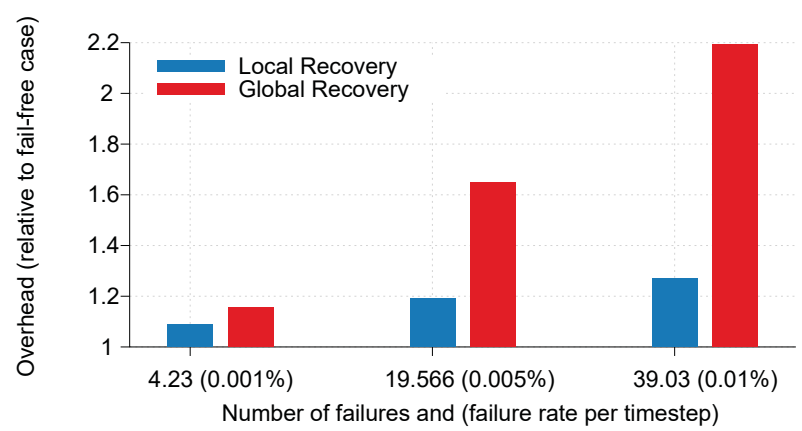

Figure 3: Recovery overheads for local and global recovery obtained from simulations based on the model for the parallel 3D stencil code running on a 4096 process grid.

to model the execution time of a 1-D stencil computation:

$$
\begin{aligned}
T(i, j) & =\max (T(i-1, j-1), T(i-1, j+1))+T_{\text {local }}+r \\
T(0, j) & =0 \\
T_{\text {local }} & =t_{1} \text { if no delay, or } t_{2} \text { if delayed }
\end{aligned}
$$

where $T(i, j)$ is the total execution time at $i$-th timestep of $j$-th process. We assume that all the processes are assigned linearly to nodes so that every node communicates with its neighbors $(j-1$ and $j+1)$. $T_{\text {local }}$ is the execution time of the local computation per timestep, which is determined based on the probability of process failure; we define two cases, $t_{1}$ and $t_{2}$ to represent no-failure (no-delay) and failure (delayed), respectively. Additionally, a random performance noise $r$ is applied in every timestep. The term, $\max (T(i-1, j-1), T(i-1, j+1))$, represents synchronization (communication) with neighboring processes; we assume that every process waits for the completion of its neighbors before starting its next timestep. This may not be true for state-of-art optimized applications, which are typically designed to compute on the interior mesh points while neighbor exchanges of ghost regions happen. Note that our model also does not consider the interconnect network performance and the checkpoint overhead. For example, the recovery from a failed process may involve allocating a spare process, which may dramatically change the network topology of the nodes around the failed ones. However, the goal of our model is to capture the execution pattern of stencil-based applications and to explore delay propagation and resulting performance impacts due to local recovery from failures. Note that, as will be shown in Section 6, results from this model do corroborate experimental results. A more rigorous model and verifications would be helpful to better understand the relationship between system performance and application behavior and for optimizing our recovery schemes, and we plan to explore it as part of our ongoing research.

\subsection{Simulation}

We have implemented a simulator using the model described above in Matlab and used it to study the progress of each process in the event of failures. The simulator computes the execution time at each core for every timestep using the recurrence presented above. The failure rates are provided as input parameters and are used to determine $t_{1}$ and $t_{2}$. These simulations do reproduce the progress of each 
timestep as presented in Figure 2. For example, the 32 core case shows how the first two delay propagations are merged. We observe similar results from the experiments using real 1D stencil code in Figures 7 and 8 presented in Section 6.5.

Next, we extended our model and simulator to model a 3D stencil (with 6 neighbors) on 4096 cores, to estimate the execution pattern of $\mathrm{S} 3 \mathrm{D}$ on a $16^{3}$ process grid for 100 timesteps. In this case, we use the set of parameters presented in the table below:

\begin{tabular}{|c|c||l|c|}
\hline$t_{1}$ & 1.0 & Failure rate (in \%) & $0.001,0.005,0.01$ \\
$t_{2}$ & 5.0 & $\mathrm{r}$ & $(0,0.1)$ \\
\hline
\end{tabular}

We ran each simulation 30 times and plot the average execution time (for each core) for local recovery and global recovery in Figure 3. In case of the global recovery model, we apply the same delay factor $t_{2}$ to all the processes when a failure occurs in one (or many) of the processes. This may be optimistic as it does not consider the potentially high overhead for MPI communicator recovery, as reported by our previous work $[15,36]$, but we do apply the same delay effect to both cases for fair comparisons. The model-based simulation shows the delay from multiple failures is masked by local recovery, reducing the recovery overhead down to $22 \%$ of the global recovery case.

\subsection{Effectiveness of Failure Masking for larger domains}

Now that we have seen how the model appropriately describes the propagation effect of the failures, we want to study the feasibility of failure masking towards extreme scales. To do that, we suppose a future exascale machine whose cores can be arranged following a 100x100x100 7point 3-D Stencil topology, totaling 1,000,000 cores. A cubic domain decomposition can be seen as the worst case scenario, because a non-cubic domain would lead to longer propagation times for the same machine size. We further assume that each core will simulate a certain cubic subdomain of size $10^{3}(1,000)$ points or $20^{3}(8,000)$ points.

Assume now that a failure happens in the center point, which represents the worst case in terms of propagation delay (i.e. a failure in the center will be the fastest to propagate to the whole domain). In this scenario, 50 cores have to be traversed (i.e. 50 hops) for the failure to reach the six walls of the domain, at which point only $17.18 \%$ of the cores have been affected. To propagate to half of the cores $(50.75 \%)$, 75 hops have to occur and to propagate to the whole domain requires 150 hops.

Therefore, assuming that the computation to advance each point one timestep requires $0.5,1$ or 5 seconds, the total propagation time to reach half of the domain will be $75 \cdot 10 \cdot\{0.5,1,5\}=\{375,750,3750\}$ seconds, respectively (1000 points per core) and $\{750,1500,7500\}$ seconds $(8000$ points per core). To propagate to the whole domain, it will take exactly double the time amounts. Since in current executions we are experimentally observing a 5 seconds iteration time, the smaller cases can serve only as lower bounds.

As we can see, all the estimates are larger than the expected exascale MTBF. Therefore, failure masking will be effective for larger domains.

\section{FENIXLR IMPLEMENTATION}

In this section, we first describe our initial attempts at implementing our approach using an MPI-based framework (i.e., ULFM) and the challenges faced. We then present the implementation FenixLR. Note that while FenixLR maintains the same programming interface as Fenix [15], it has been built from scratch on top of Cray's uGNI interface the only component re-used from Fenix is the checkpointing module.

Also note that using local rollback and global continue results in increased complexity as compared to global rollback. When using global rollback, all threads are restarted from a known previous consistent state and all lost computation and communication have to be repeated. As a result, there is no need for the respawned threads to be distinguished from the surviving ones, since all the recovery actions will be the same in all threads.

However, when using local rollback and global continue, the respawned threads may need to receive messages from the non-failed threads that were already sent. As a result, there has to be a mechanism for the respawned threads to request these messages (since the non-failed threads did not rollback).

\subsection{Experiences with MPI-based implementa- tions}

A key challenge faced in our MPI-based implementations (using ULFM) was the cost of the ULFM Comm_shrink operation as implemented in the ULFM prototype. We did not find any conceptual problems with ULFM, instead, the prototype implementation was not robust enough. In our experimentation, we found that the cost of ULFM's communicator shrink increased worse than linearly with the system size, and took a non trivial amount of time to complete. As a result we explored two options to avoid using it.

Option 1: No communicator repair. The ULFM specification suggests that a failure can be acknowledged and the communicator should be usable again for point to point communications (i.e., no collectives) without the need to repair (i.e., shrink) it, as long as no contact is made to the failed rank. Our first implementation was based on this specification. After implementing a benchmark for it (1D PDE), we found out that MPI operations on a non-repaired failed communicator were unpredictable. Most of the times the runtime was unsuccessful in delivering point to point communications after a process failure.

Option 2: Many communicators with only two ranks. After trying to solve the aforementioned problem, and given that ULFM is the only MPI runtime that survives real failures, we changed our strategy. Given that, in our targeted application, each rank only communicates with a constant number of neighbors (independent of the system size), we created a layout of "disposable communicators", each of which only contained two ranks. In other words, assuming no collectives were needed, we created a communicator for every pair of ranks that had to communicate. The idea behind this approach was, upon failure, to allow the disposal of all communicators that were associated with the failed rank. In order to recover from the failure, all the compute ranks had to be connected with all spare ranks that can be used for recovery. As this would create a non-scalable number of communicators in the spare ranks side, we divided the domain in groups containing a constant number of compute ranks, and also associating a constant number of spare ranks to each group. The main problem with this design is 
that the application has to understand the specific details of the recovery procedure, and all possibilities in terms of the order of execution upon failure have to be covered (e.g., did the failed thread issue an MPI_Send or MPI_Recv to be matched by neighbor $A$ in the future? Was $A$ more advanced in the execution than the failed thread? etc.). All these possibilities were implemented in the one-dimensional version of the PDE solver. However, it was a non-trivial task to understand all the situations and design a work around them, as was clearly not feasible for higher dimensions.

As a result, we implemented our prototype of FenixLR directly on top of uGNI, avoiding having an MPI runtime altogether, and only implemented the subset of MPI operations used by S3D. We understand that MPI-based frameworks in general and ULFM specifically are evolving rapidly and we will revisit them once the above issues are fixed.

\subsection{Implementation Overview}

FenixLR has been designed in a modular and layered manner. Its architecture is composed of four key modules: communication logic, data resiliency, process resiliency, and transport layer. The communication module is object oriented in order to isolate failure recovery mechanisms from the different communication protocols. A base class Command offers an abstraction for reliably requesting services or communicate with other processes using the chosen transport layer. All communication mechanisms (i.e., checkpoint, send/recv, barrier, broadcast, etc.) rely on this class and implement the logic in a simple manner without including any fault tolerance code. Data resiliency implements the checkpointing and data recovery mechanisms, which can be substituted by an interface to any existing checkpointing library. Process resiliency manages the spare process pool and implements the protocol to restart processes. The transport layer has been implemented using uGNI, but could be easily extended to other transport APIs. Communications in production versions of MPI implementations are probably faster than in our framework, because we have not optimized the performance of data transfer yet. Specifically, we cannot use RDMA in uGNI due to inconsistent behaviors of the network driver that appears only after injected failures. Under normal operation, when a failure occurs, the runtime core invokes the different modules, orchestrates the recovery process at high level, and determines which commands have to be re-executed.

Each rank maintains a set of ranks with whom it is connected. To start a new communication from rank $P_{1}$ to $P_{2}$, a handshake process is used to logically connect both ranks (add a table entry on both sides). If process $P_{i}$ fails, a spare process $S$ replaces $P_{i}$ and re-creates the handshake with the processes $P_{i}$ was communicating with. Then, a background collective is used to scatter a failure notification. If a thirdparty process $P_{3}$ tries to connect to a failed process $P_{i}$ before the scattered notification reached it, it will detect the failure and try to contact the first spare process available, which will notify that the failed process $P_{i}$ has already been substituted by $S$. A node failure is viewed as a set of process failures.

FenixLR offers three language bindings: $\mathrm{C}, \mathrm{C}++$ and Fortran. We maintained the same programming interface as Fenix [15], as we had demonstrated its low programming overhead - it required less than 35 new, changed, or rearranged lines of code for $\mathrm{S} 3 \mathrm{D}$, (see Section IV.B in [15]). In fact programming local recovery using FenixLR is even simpler than programming global recovery, because the status returned by Fenix_Init () can take three different values for global recovery (i.e., New, Respawned, or Survivor) and only two values for local recovery - survivor status is no longer valid, since all survivor processes do not get interrupted from their processing. This simplifies the application side of the recovery logic.

\section{EVALUATION}

In this section we present an experimental evaluation of the effectiveness and performance of our failure masking and local recovery techniques implemented in FenixLR using the S3D combustion simulation on the Titan Cray XK7 at ORNL. Specifically, we experimentally show that our local recovery algorithm can tolerate multiple failures and can mask the total recovery overhead so that it is comparable to that of a single failure, i.e., we empirically show that, with local recovery, the total overhead $O_{N}$ of recovering from $N$ failures is not necessarily $N \times O_{1}$, where $O_{1}$ is the overhead of recovering from a single failure.

Our previous work [15] had shown that Fenix can tolerate failures in an online and global manner with failures occurring every 47 seconds. The implementation used in that work leveraged MPI-ULFM to detect failures and recover from them. In the implementation presented in this paper, we have reduced the sources of overheads and eliminated ULFM dependencies, and we have reimplemented the fault tolerance mechanisms on top of uGNI, Cray's interconnect API. We experimentally evaluate how these algorithmic improvements can enable FenixLR to effectively handle even higher failure rates (i.e. every 5 seconds).

In summary, the goals of the experimental evaluation presented in this section are to demonstrate that (i) using the failure masking and local recovery techniques presented in this paper, FenixLR can enable tightly-coupled stencil-based applications such as S3D, to recover from node failures occurring as frequently as every 5 seconds, (ii) the failure recovery is scalable, and (iii) the overhead of recovering from failures is not proportional to the system size.

As mentioned, all the experiments were performed on the Cray XK7 Titan at ORNL. Titan is composed of 18688 16core CPUs and the same number of GPUs. Every pair of nodes is connected to a single custom system-on-chip Gemini ASIC network interconnect. Gemini ASICs are connected using a 3D torus topology. Applications can directly access network capabilities using uGNI, the user level proprietary interface from Cray, which is forward compatible with newer versions of Cray networks, such as Aries.

In what follows, we present our experimentation methodology and describe the experiments in detail.

\subsection{Methodology}

A key goal of our evaluation is to study how the presented approach behaves at current scales, and use this to try to explore behaviors and performance at exascale. As a result, we have conducted our experiments on up to 262272 cores.

In our experiments we first evaluate the scalability of the checkpointing technique implemented in FenixLR using S3D with a checkpoint size of $130 \mathrm{MB} /$ core. We then study the overheads related to the recovery process and its scalability. In this experiment, we inject worst-case failures, i.e., sets of failures that do not allow recovery propagation delays to 


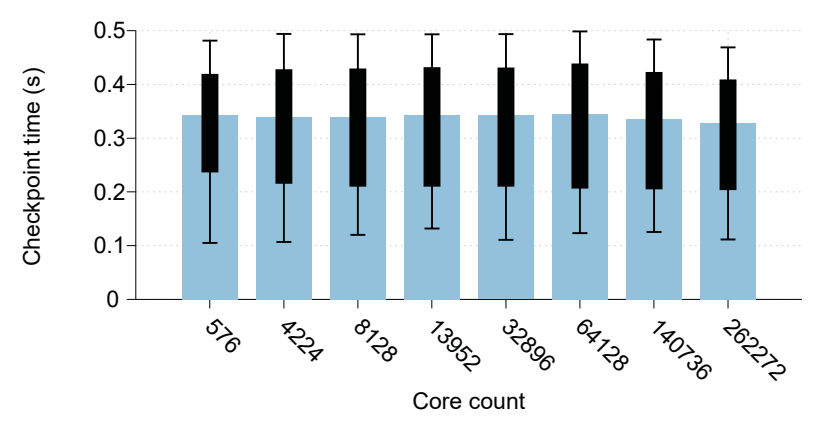

Figure 4: Weak scaling of checkpointing using FenixLR with a checkpoint size of $130 \mathrm{MB} /$ core.

merge and, therefore, the total overhead is the sum of the recovery overhead for each failure. We show that FenixLR handles frequent node failures, i.e., MTBFs as low as 5 seconds, with total overheads of up to $50 \%$ in the worst case scenario. The results empirically show that this method can be more efficient than theoretical full redundancy, for the targeted class of applications.

Finally, we show the benefits of FenixLR's local recovery capabilities using S3D on up to 140736 cores $(140608+128$ spare cores). We demonstrate how in real scenarios, local recovery can mask failures, i.e., result in a total overhead that is comparable to one failure recovery, regardless the number of failures.

In order to perform these experiments we inject node failures. Node failures are injected by simultaneously sending SIGKILL signals to all application processes running on that node. As the network setup parameters are stored in process memory, when killing the process we do not allow any disconnections - this is consistent with the behavior if a real failure occurred. The processes on the other nodes get error codes when trying to perform a uGNI operation with the processes that are killed. In this sense, we say that we inject real failures, as opposed to pretending that a process has failed. In what follows, when we talk about failures, we refer to node failures, which is equivalent to $N$-process failures, where $N$ is the total number of processes on a system node, blade, etc. By default, the experiments use $N=16$. Unless specified otherwise, all tests have been repeated 5 times. Error bars in all Figures show the average, first quartile, third quartile, maximum and minimum of the 5 repetitions.

We only display the experiments with MTBFs lower than a minute due to the fact that higher MTBFs (such as 5 minutes, 10 minutes or 30 minutes, etc.) show negligible recovery overheads relative to the total execution time.

\subsection{Checkpoint Scalability}

In [15] we evaluated double in-memory checkpoints and demonstrated that it scales independently of the number of processes. In this paper, we reevaluated the checkpoint scalability using the FenixLR framework, while also injecting failures and with up to 16 times larger data sizes per core. Figure 4 plots the weak scalability of checkpointing up to 262272 cores (including 128 spare cores) using a larger checkpoint size of $130 \mathrm{MB} /$ core. In this experiment, failures were injected every 10 seconds. As we can see from this plot, the overhead due to checkpointing remains the same independent of the number of cores, which demonstrates very

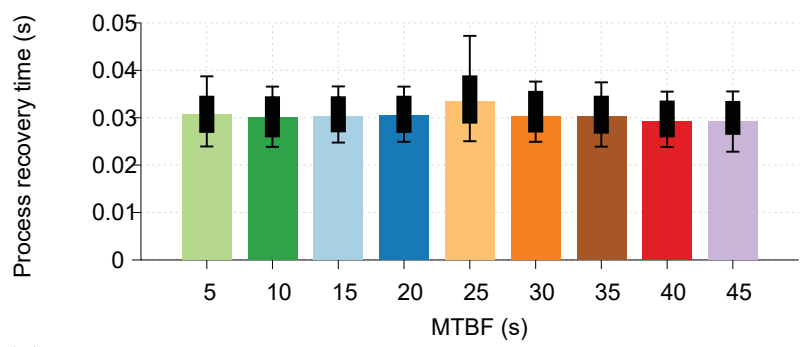

(a) Different MTBFs. Core count fixed at 4736 cores (4096 compute cores and 640 spare cores).

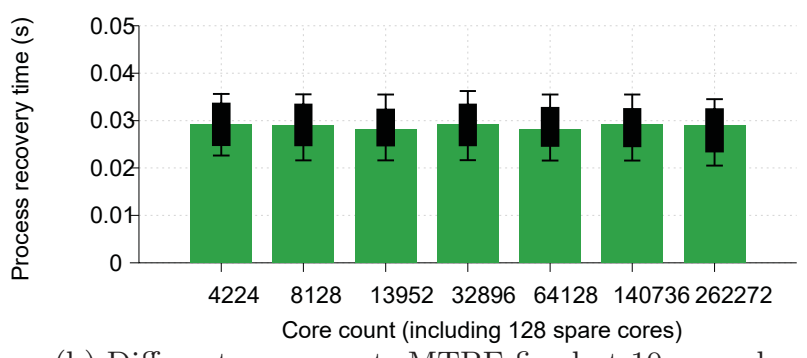

(b) Different core count. MTBF fixed at 10 seconds.

Figure 5: Average process recovery overhead.

good weak scalability.

\subsection{Recovery time for different MTBFs}

The goal of this second experiment is to demonstrate that FenixLR is capable of handling high-frequency failures occurring up to every 5 seconds. In this experiment we also study the overheads due to process recovery and empirically demonstrate that the performance of FenixLR does not depend on the total number of processes (i.e., demonstrates good scalability), which is highly desirable at exascale. We performed these experiments using S3D on 4736 cores (4096 compute and 640 spare cores), unless otherwise specified.

Our goal in this experiment is also to explore the benefits of local recovery over global recovery. Consequently, in this experiment we engineer the set of failures injected so that they do not allow propagation delays due to local recovery to merge, and therefore the total overhead is the sum of the recovery overhead for each failure. In other words, this experiment evaluates the worst-case local recovery overhead. Process recovery overhead. First, we study the total overhead of recovering from a failure for different failure frequencies. Figure 5(a) plots the average overhead of process recovery for different frequencies of node failure injected during an S3D execution of about 200 seconds. Each bar represents the average time to recover the processes from a failure. This overhead is initially seen only in the spare processes that substitute the failed ones, and then propagates to the rest of the domain due to the communication pattern of the application. This figure does not include overheads due to data recovery (i.e., fetching the checkpoint). Note that fetching the checkpoint is the exact inverse process of checkpointing: First, the checkpoint has to be fetched from the neighbor that stores it in-memory to the memory of the spare process. Then, it has to be copied using memcpy() to the application memory. Therefore, the overhead of fetching the checkpoint will be similar to the checkpointing overhead. 
Scalability. Figure 5(b) plots the average process recovery time for every failure. The figure demonstrates that, for our experiments on up to 262272 cores, the local recovery overhead is constant and independent of the number of processes in the system. These experiments were performed with a fixed MTBF of 10 seconds, and all the experiments used 128 spare processes. Furthermore, experiments for system sizes smaller than $64 \mathrm{k}$ have been repeated with a different total number of node failures, ranging from 1 to 8 node failures, and the results have been averaged in the plots.

\subsection{Total overhead for different MTBFs}

The previous experiments demonstrated that the process recovery time for a single failure is small, and is constant and independent of the total number of cores used by the application as well as the frequency of failures. In the next set of experiments, we study the total overhead due to fault tolerance, i.e., including overheads due to checkpointing, process/data recovery and rollback. The goal of these experiments is to compare the end-to-end execution time of a failure-free, checkpoint-free execution with the end-to-end execution time with different MTBFs. The experiment is run using a fixed core count of 4096 cores and 640 spare processes and a checkpoint size of $53 \mathrm{MB} /$ core.

Figure 6 plots the results of the experiment. For different failure rates ranging from 5 to 45 seconds, Figure 6 plots the total overhead relative to a failure-free, checkpoint-free execution base case. The total number of failures ranges from 48 processes ( 3 nodes) to 528 processes (33 nodes), as noted on top of each bar, during a total time of about 150 seconds.

The right-most bar in the figure shows the overhead of the same experiment but using global recovery while injecting failures every 47 seconds, as described in [15]. We can see that by using local recovery we obtain much lower performance penalties even at higher failure rates. For example, note that local recovery allows failures every 20 seconds with an overhead below $25 \%$, while global recovery allows failures every 47 seconds $(\sim 2.35 \mathrm{x})$ with an overhead of $31 \%$ $(\sim 1.25 \mathrm{x})$. Also note that the total overhead of running the experiment in the worst-case scenario (i.e., with node failures every 5 seconds), is $51 \%$. That is, it is slightly worse (i.e., $1 \%$ worse) than the theoretical best-case overhead of using 2-way redundancy. Again, it is important to emphasize that in this experiment, we injected worst-case failures, i.e., in which failure masking does not occur in most cases - the only exception is the 5-s MTBF experiment, in which the total time is just a small portion above the 10-s MTBF test.

\subsection{Local Recovery with Failure Masking}

The goal of this last experiment is to show that, with local recovery, the total overhead $O_{N}$ of recovering from $N$ failures is not necessarily $N \times O_{1}$ (being $O_{1}$ the overhead of recovering from a single failure). This is what we term as failure masking.

Experiments using a 1D PDE. We will begin our experiments by studying the behavior of local recovery for a 1-dimensional stencil-based Partial Differential Equation (PDE) solver, or 1D PDE for short. Figure 7 shows several executions of 1D PDE on 32 compute cores and 4 spare cores with different number of failures. These figures are based on results obtained from actual runs on Titan (Cray

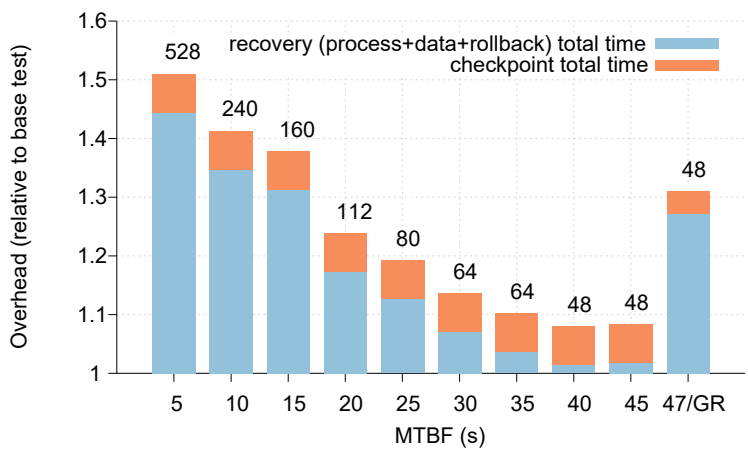

Figure 6: Comparison of the total overhead due to failures and fault tolerance mechanisms with different MTBFs. Core count fixed at 4736 cores (4096 cores and 640 spare ranks). Checkpoint size is $53 \mathrm{MB} /$ core. Numbers on top of the bars indicate the total number of process failures injected during the execution.

XK7 at ORNL) during which we injected real process failures as described above. The $\mathrm{X}$ axis in the plots represents the core number (or rank number), and the $\mathrm{Y}$ axis represents wall clock time. Each rank simulates a certain number of points in the 1-D domain. Adjacent ranks operate on adjacent spans of the 1-D domain. Each horizontal line in a plot represents an iteration (i.e., every time the solver communicates with the neighbors in order to advance the solution). Red crosses represents injected process failures. A straight line means that all processes complete the iteration at the same physical time. When a failure occurs, the recovery delay does not get propagated immediately to the entire domain, but, instead, the neighbor processes that are immediately adjacent to the recovered process are the first to be delayed. This delay propagates out to their neighbors in the next iteration, and their neighbor's neighbors in the following iteration, and so on until the entire domain is eventually covered. Note how 2, 3 and 4 failure cases shown in Figures 7(b), 7(c) and 7(d) have a similar recovery overhead as the one failure case, i.e., Figure $7(\mathrm{a})$.

Figure 8 shows a longer experiment using a larger number of processes and injecting node failures - 13984 total cores, including 13824 compute cores and 160 spare cores. As we can observe, in this case the delay propagation waves caused by the failures do not merge. As a result, in this case the total time to solution only increases by the time to recover from a single failure, independent of the total number of failures.

By comparing Figure 7 with Figure 2 (left) and Figure 8 with Figure 2 (right), we can see that results from the model and simulation accurately predict the real experimental results. This implies that the presented discrete event simulator does capture the benefit of local recovery.

The results presented above not only validate our algorithm and implementation, but also demonstrate that local recovery can be beneficial in extreme scale environments, where high-frequency failures are expected. The results also demonstrate that local recovery is a scalable approach, both in the number of failures and the size of the system.

Experiments using S3D. Figure 9 shows the behavior of local recovery for the S3D Stencil 3-D PDE solver. Each line in this figure represents a timestep, and the color of the line 


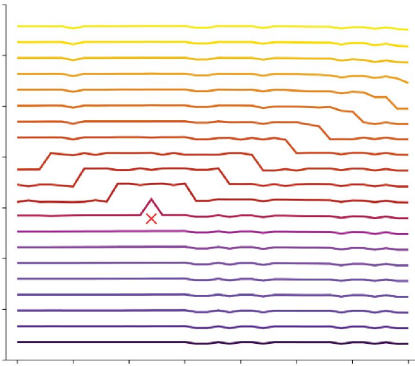

(a) One process failure

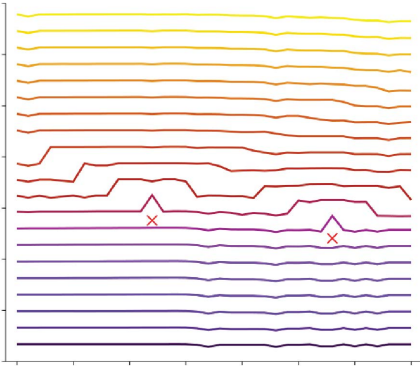

(b) Two process failures

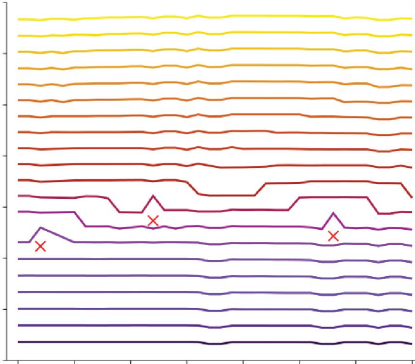

(c) Three process failures

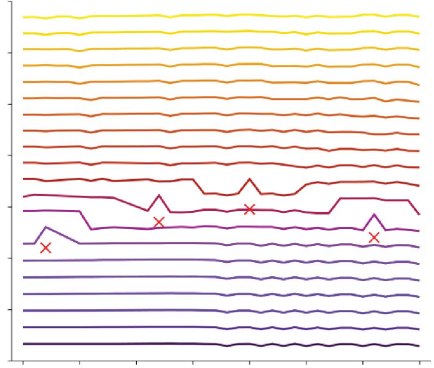

(d) Four process failures

Figure 7: Behavior of local recovery for a 1D PDE using 36 cores (32 compute cores and 4 spare cores). X axis represents process number (or rank) and Y axis indicates wallclock time. Each line in a figure represents a timestep, and the color of the line represents how advanced the simulation is (i.e., it advances from yellow to dark purple). Each red ' $\mathrm{X}$ ' represents a failure. A straight line means all processes compute the timestep at the same physical time. When a failure occurs, the recovery delay does not get propagated immediately to the entire domain. Instead, the immediately adjacent neighbor processes are the first to be delayed, which in turn propagate the delay to their immediate neighbors, resulting in the delay eventually spanning across the entire domain.

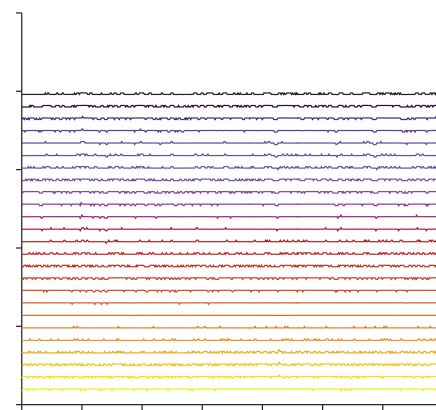

(a) Base execution

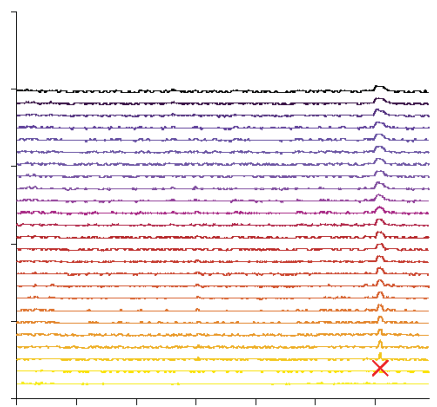

(b) One node failure

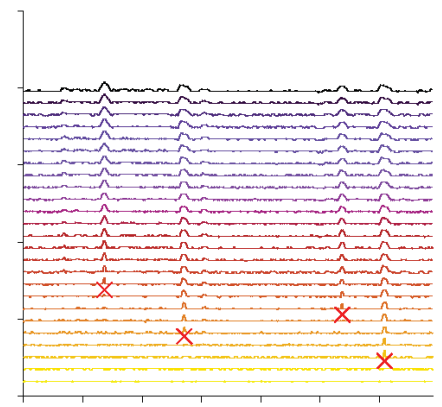

(c) Four node failures

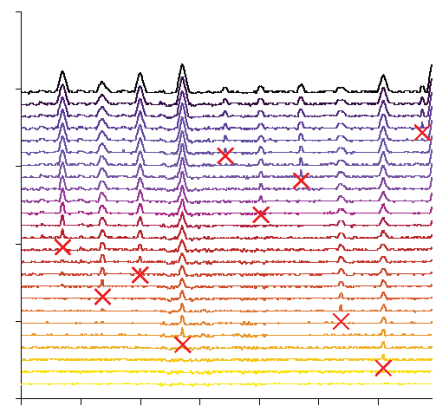

(d) Ten node failures

Figure 8: Behavior of local recovery for a 1D PDE using 13984 cores (13824 compute cores and 160 spare cores), with failures injected every 10 seconds.

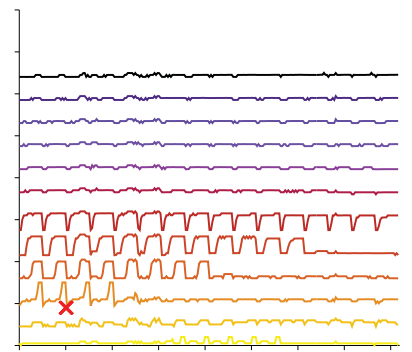

(a) 4224c $1 \mathrm{f}$

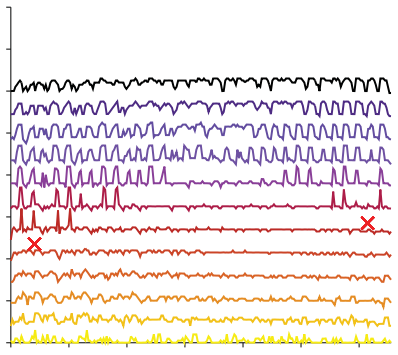

(e) $32896 \mathrm{c} 2 \mathrm{f}$

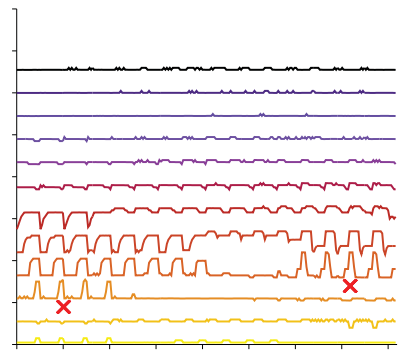

(b) 4224c $2 \mathrm{f}$

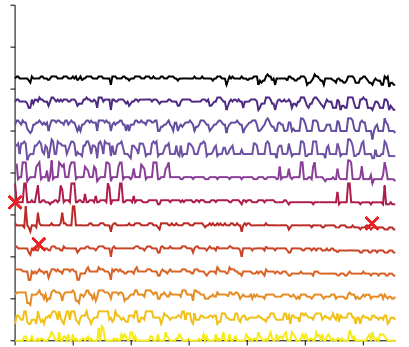

(f) $32896 \mathrm{c} 3 \mathrm{f}$

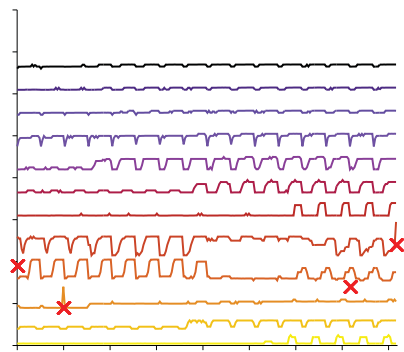

(c) $4224 \mathrm{c} 4 \mathrm{f}$

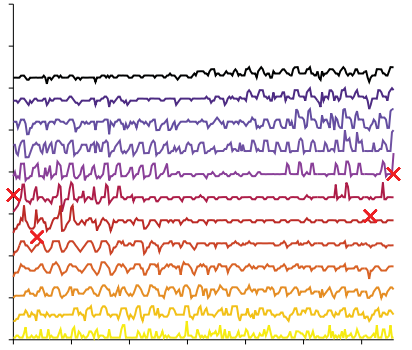

(g) 32896c 4f

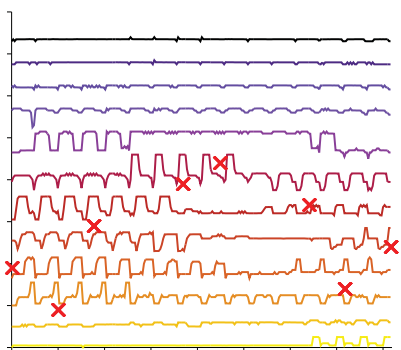

(d) $4224 \mathrm{c} 8 \mathrm{f}$

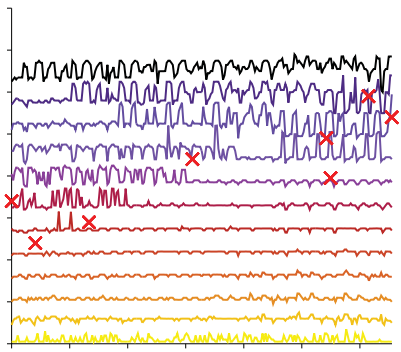

(h) $32896 \mathrm{c} 8 \mathrm{f}$

Figure 9: Behavior of local recovery for the S3D Stencil 3-D PDE solver for different core counts and number of failures. 
represents how advanced the simulation is - it advances from yellow to dark purple. The $\mathrm{X}$ axis in the plots represents the rank number, which is linearly mapped to the 3D S3D domain. This mapping between $3 \mathrm{D}$ space and $1 \mathrm{D}$ ranks is done in a straightforward manner - beginning at point $(0,0,0)$ we assign rank numbers by counting first in the $\mathrm{Z}$ direction, followed by the $\mathrm{Y}$ direction and finally in the $\mathrm{X}$ direction. The $\mathrm{Y}$ axis represents wall clock time. In the caption for each plot, a 'c' refers to cores and an ' $\mathrm{f}$ ' refers to the number of node failures injected. Note how the overheads in the first three columns are the same, which empirically demonstrates how failure masking works. The plots in these figures follow the same format as Figure 7. In the 3D case, however, the communication pattern between ranks is not as obvious in the plots as in the $1 \mathrm{D}$ case due to the mapping for ranks to the 3D domain. Stencil-based communications in the 3D domain proceed as follows: each process (except along the edges) communicates with its six neighboring processes in the up/down, front/back and left/right directions.

Figure 10 summarizes the relative costs of recovering from different numbers of failures (from 1 to 8 ) at different scales (512, 4096, 8000, 13824, 32768, 64000 and 140608 cores). The costs are normalized by the cost of recovering from one failure. The plots show that in many cases, the total overhead is close to that for the 1 failure case. Specifically, the increase in overheads due to multiple failures is around $2 \%$ or lower. This difference can be due to different rollback overheads. If the failures occur right after a checkpoint, the rollback overhead will be smaller. However, if the failures occurs right before a checkpoint, the rollback overhead will be much larger, because almost an entire iteration will have to be recomputed. However, there are also cases where the recovery overhead increases by more than $2 \%$, up to 6 or $12 \%$. In these cases, there was at least one failure that occurred within the propagation delay resulting from the recovery of a previous failure. This is consistent with Figure 9. For example, if we compare Figure 9(d) with the rest of the experiments using 4224 cores, we observe a large difference in the total overhead. This difference is due to a failure occurring in a core, which has already experienced a delay due a previous failure in a neighboring core. In this specific case, the core with the third failure (counting from the left) has experienced a delay due to the second failure.

The results presented in this section have demonstrated how local recovery enables failure masking, which is also highly desirable at extreme-scales. Specifically, we have empirically demonstrated that, with local recovery, the total overhead $O_{N}$ of recovering from $N$ failures is not necessarily $N \times O_{1}$ (being $O_{1}$ the overhead of recovering from a single failure).

\section{CONCLUSION}

In this paper we have explored an approach for failure masking and online, local recovery from high-frequency node failures for stencil-based parallel applications, using application guided checkpointing as a means for data resilience. The approach is based on understanding the propagation of delays associated with local recovery for stencil-based computation/communication pattern, and the observation that the impact of the delay associated with multiple failures is often not additive, allowing this approach to be feasible and scalable. In this paper we first used simulations to validate the approach and then design and implement FenixLR

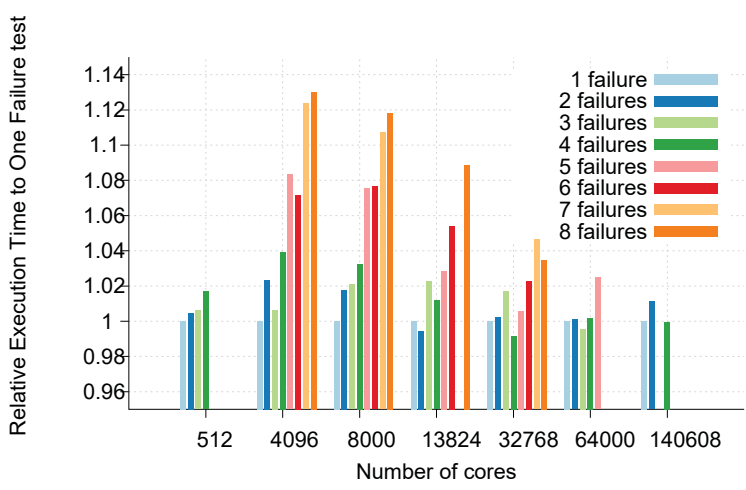

Figure 10: Summary of the relative costs of recovering from different numbers of failures (from 1 to 8 ) at different scales (512, 4096, 8000, 13824, 32768, 64000 and 140608 cores). The costs are normalized by the cost of recovering from one failure. Note that for the $64 \mathrm{k}$ and $140 \mathrm{k}$ core cases, not all the scheduled experiments made it through Titan's execution queue, and only those experiments that made it through are plotted. For the $262 \mathrm{k}$ core case, only one experiment went through the execution queue, and so no comparison is available.

framework from scratch. We then deployed it on the Titan Cray XK7 production system at ORNL and used it to enable failure masking and local recovery for the S3D combustion application, which is a key application for exascale [2]. The simple FenixLR local recovery interface enabled this integration to be achieved with less than 35 lines of S3D code to be added, changed, or rearranged.

We also presented an extensive experimental evaluation of the local recovery algorithms in FenixLR on Titan using $\mathrm{S} 3 \mathrm{D}$, while injecting real failures. Our experiments demonstrate that local recovery in FenixLR provides a viable solution for stencil-based applications, for addressing node failures occurring as frequently as every 5 seconds, on scales up to 262144 cores. Our results show an overall performance overhead of $13.75 \%$ while tolerating node failures every 30 seconds (worst-case scenario, as the MTBF for the exascale systems is expected in order of minutes [13]). We also show that this overhead is smaller as compared to the overheads of using global recovery techniques previously presented on Fenix [15], and is also smaller than the overhead of traditional checkpointing with global offline restart that is currently used by $\mathrm{S} 3 \mathrm{D}$ for its large-scale production runs on Titan.

Our experiments also evaluated the scalability of the local recovery stages in FenixLR, and demonstrate that all fault tolerance aspects scale well with increasing number of cores. Finally, our experiments also demonstrated how local recovery enables failure masking, i.e., the overheads on the total execution time due to recovery from multiple failures is comparable to that of only one failure.

The results presented in this paper open a new discussion about whether power-hungry hardware reliability mechanisms are needed or not. For example, while error correcting codes (ECC) found in the main memory and cache are known to help correct memory failures as often as every few seconds in current large-scale systems [33], they are accounted for more than $20 \%$ of the power consumption of the memory subsystem [35]. In this paper we present an im- 
plementation of the correct combination of techniques that enable constant scalability and small overhead recovery, and show how frequent failures (i.e. down to MTBFs of 5 seconds) can be tolerated while masking its overhead. This can translate into a co-design question: can we have supercomputers made of less-reliable hardware ${ }^{1}$ able to run Stencillike applications with similar time, but with reduced power consumption? In such case, our approach could be applied by simply considering the actual failure a fail-stop process failure. We leave the study of this power-resilience tradeoff for future work.

Our ongoing and future work includes (1) exploring how the conclusions in this paper apply to other classes of applications (beyond stencil-based), (2) modeling and understanding optimal sizes for ghost regions so that delays due to local recovery propagate slower, and (3) studying how asynchronous transfers of checkpoints can reduce checkpointing overheads without impacting simulation time.

\section{Acknowledgments}

The authors would like to thank Josep Gamell, Robert Clay and George Bosilca for interesting discussions related to this work. The research presented in this work is supported in part by National Science Foundation (NSF) via grants numbers ACI 1339036, ACI 1310283, CNS 1305375, and DMS 1228203, by the Office of Advanced Scientific Computing Research, Office of Science, of the US Department of Energy through the SciDAC Institute for Scalable Data Management, Analysis and Visualization (SDAV) under award number DE-SC0007455, RSVP award via subcontract number 4000126989 from UT Battelle, the ASCR and FES Partnership for Edge Physics Simulations (EPSI) under award number DE-FG02-06ER54857, and the ExaCT Combustion Co-Design Center via subcontract number 4000110839 from UT Battelle. The research at Rutgers was conducted as part of the Rutgers Discovery Informatics Institute $\left(\mathrm{RDI}^{2}\right)$.

Sandia National Laboratories is a multi-program laboratory managed and operated by Sandia Corporation, a wholly owned subsidiary of Lockheed Martin Corporation, for the U.S. Department of Energy's National Nuclear Security Administration under contract DE-AC04-94AL85000.

\section{REFERENCES}

[1] S. Amarasinghe and et al. ExaScale Software Study: Software Challenges in Extreme Scale Systems. Technical report, DARPA IPTO, Air Force Reserach Lab, Sept. 2009.

[2] S. Amarasinghe and et al. Exascale Programming Challenges. In Proceedings of the Workshop on Exascale Programming Challenges, Marina del Rey, $C A, U S A$. U.S Department of Energy, Office of Science, Office of Advanced Scientific Computing Research (ASCR), Jul 2011.

[3] L. Bautista-Gomez, S. Tsuboi, D. Komatitsch, F. Cappello, N. Maruyama, and S. Matsuoka. FTI: High Performance Fault Tolerance Interface for Hybrid Systems. In Proceedings of International Conference for High Performance Computing, Networking, Storage and Analysis, SC 2011, 2011.

\footnotetext{
${ }^{1}$ Or hardware that can turn on and off power-hungry re-
} silience hardware capabilities, like memory ECC.
[4] P. Beckman, R. Brightwell, B. R. de Supinski, M. Gokhale, S. Hofmeyr, S. Krishnamoorthy, M. Lang, B. Maccabe, J. Shalf, and M. Snir. Exascale Operating Systems and Runtime Software Report. Technical report, US Department of Energy, December 2012.

[5] W. Bland, G. Bosilca, A. Bouteiller, T. Herault, and J. Dongarra. A Proposal for User-Level Failure Mitigation in the MPI-3 Standard. Technical report, Innovative Computing Laboratory, University of Tennessee, February 2012.

[6] W. Bland, A. Bouteiller, T. Herault, G. Bosilca, and J. J. Dongarra. Post-failure recovery of MPI communication capability: Design and rationale. International Journal of High Performance Computing Applications, 2013.

[7] A. Bouteiller, T. Herault, G. Krawezik, P. Lemarinier, and F. Cappello. MPICH-V Project: A Multiprotocol Automatic Fault-Tolerant MPI. International Journal of High Performance Computing Applications, 20(3):319-333, 2006.

[8] A. Bouteiller, T. Ropars, G. Bosilca, C. Morin, and J. Dongarra. Reasons for a pessimistic or optimistic message logging protocol in MPI uncoordinated failure, recovery. In IEEE International Conference on Cluster Computing and Workshops, CLUSTER 2009, pages 1-9, 2009.

[9] B. Bouteiller, P. Lemarinier, K. Krawezik, and F. Cappello. Coordinated checkpoint versus message log for fault tolerant MPI. In Proceedings of the IEEE International Conference on Cluster Computing, pages 242-250, 2003.

[10] K. M. Chandy and L. Lamport. Distributed snapshots: determining global states of distributed systems. ACM Trans. Comput. Syst., 3(1):63-75, Feb. 1985.

[11] J. H. Chen, A. Choudhary, B. de Supinski, M. DeVries, E. R. Hawkes, S. Klasky, W. K. Liao, K. L. Ma, J. Mellor-Crummey, N. Podhorszki, R. Sankaran, S. Shende, and C. S. Yoo. Terascale direct numerical simulations of turbulent combustion using S3D. Computational Science and Discovery, 2(1):015001, Jan. 2009.

[12] C. Coti, T. Herault, P. Lemarinier, L. Pilard, A. Rezmerita, E. Rodriguez, and F. Cappello. Blocking vs. Non-Blocking Coordinated Checkpointing for Large-Scale Fault Tolerant MPI. In ACM/IEEE Proceedings of the International Conference on High Performance Computing, Networking, Storage and Analysis, pages 18-18, Nov 2006.

[13] J. Dongarra and et al. The International Exascale Software Project Roadmap. International Journal of High Performance Computing Applications, 25(1):3-60, 2011.

[14] E. N. M. Elnozahy, L. Alvisi, Y.-M. Wang, and D. B. Johnson. A survey of rollback-recovery protocols in message-passing systems. ACM Comput. Surv., 34(3):375-408, Sep 2002.

[15] M. Gamell, D. S. Katz, H. Kolla, J. Chen, S. Klasky, and M. Parashar. Exploring Automatic, Online Failure Recovery for Scientific Applications at Extreme Scales. In Proceedings of the International Conference on High Performance Computing, Networking, Storage and Analysis, SC '14, 2014. 
[16] M. Gamell, K. Teranishi, M. A. Heroux, J. Mayo, H. Kolla, J. Chen, and M. Parashar. Exploring Failure Recovery for Stencil-based Applications at Extreme Scales. In The 24th International ACM Symposium on High-Performance Parallel and Distributed Computing, HPDC '15, June 2015.

[17] A. Guermouche, T. Ropars, E. Brunet, M. Snir, and F. Cappello. Uncoordinated Checkpointing Without Domino Effect for Send-Deterministic MPI Applications. In IEEE International Parallel and Distributed Processing Symposium (IPDPS), pages 989-1000, 2011.

[18] P. H. Hargrove and J. C. Duell. Berkeley Lab Checkpoint/Restart (BLCR) for Linux clusters. In Journal of Physics: Conference Series, volume 46, page 494. IOP Publishing, 2006.

[19] M. A. Heroux. Toward Resilient Algorithms and Applications. In Proceedings of the 3rd Workshop on Fault-tolerance for HPC at Extreme Scale, FTXS 2013, pages 1-2, New York, NY, USA, 2013. ACM.

[20] J. Hursey. Coordinated checkpoint/restart process fault tolerance for MPI applications on HPC systems. PhD thesis, Indiana University, Indianapolis, IN, USA, 2010. AAI3423687.

[21] J. Hursey, T. I. Mattox, and A. Lumsdaine. Interconnect agnostic checkpoint/restart in Open MPI. In Proceedings of the 18th ACM international symposium on High Performance Distributed Computing, HPDC 2009, pages 49-58, New York, NY, USA, 2009. ACM.

[22] J. Hursey, J. Squyres, T. Mattox, and A. Lumsdaine. The Design and Implementation of Checkpoint/Restart Process Fault Tolerance for Open MPI. In IEEE International Parallel and Distributed Processing Symposium, pages 1-8, 2007.

[23] D. Ibtesham, D. Arnold, P. Bridges, K. Ferreira, and R. Brightwell. On the Viability of Compression for Reducing the Overheads of Checkpoint/Restart-Based Fault Tolerance. In 41st International Conference on Parallel Processing (ICPP), pages 148-157, 2012.

[24] T. Islam, K. Mohror, S. Bagchi, A. Moody, B. De Supinski, and R. Eigenmann. MCREngine: A scalable checkpointing system using data-aware aggregation and compression. In International Conference for High Performance Computing, Networking, Storage and Analysis, SC 2012, pages 1-11, Nov 2012.

[25] S. Kannan, A. Gavrilovska, K. Schwan, and D. Milojicic. Optimizing Checkpoints Using NVM as Virtual Memory. In IEEE 27th International Symposium on Parallel Distributed Processing, pages 29-40, May 2013.

[26] D. S. Katz, J. Daly, N. DeBardeleben, M. Elnozahy, B. Kramer, L. Lathrop, N. Nystrom, K. Milfeld, S. Sanielevici, S. Cott, and L. Votta. Fault Tolerance for Extreme-Scale Computing Workshop, Albuquerque, NM - March 19-20, 2009. Technical Report ANL/MCS-TM-312, Argonne National Laboratory, December 2009.

[27] A. Moody, G. Bronevetsky, K. Mohror, and B. R. d. Supinski. Design, Modeling, and Evaluation of a Scalable Multi-level Checkpointing System. In
Proceedings of the ACM/IEEE International Conference for High Performance Computing, Networking, Storage and Analysis, SC 2010, pages 1-11, Washington, DC, USA, 2010. IEEE Computer Society.

[28] X. Ouyang, S. Marcarelli, and D. K. Panda. Enhancing Checkpoint Performance with Staging IO and SSD. In Proceedings of the International Workshop on Storage Network Architecture and Parallel I/Os, SNAPI 2010, pages 13-20, Washington, DC, USA, 2010. IEEE Computer Society.

[29] X. Ouyang, R. Rajachandrasekar, X. Besseron, H. Wang, J. Huang, and D. K. Panda. CRFS: A lightweight user-level filesystem for generic checkpoint/restart. In International Conference on Parallel Processing (ICPP), pages 375-384. IEEE, 2011.

[30] R. Rajachandrasekar, A. Moody, K. Mohror, and D. K. D. Panda. A $1 \mathrm{~PB} / \mathrm{s}$ file system to checkpoint three million MPI tasks. In Proceedings of the 22nd international symposium on High-performance parallel and distributed computing, HPDC 2013, pages 143-154, New York, NY, USA, 2013. ACM.

[31] T. Ropars, T. V. Martsinkevich, A. Guermouche, A. Schiper, and F. Cappello. SPBC: Leveraging the Characteristics of MPI HPC Applications for Scalable Checkpointing. In Proceedings of the International Conference for High Performance Computing, Networking, Storage and Analysis, SC 2013, pages 8:1-8:12, New York, NY, USA, 2013. ACM.

[32] K. Sato, A. Moody, K. Mohror, T. Gamblin, B. R. d. Supinski, N. Maruyama, and S. Matsuoka. Fmi: Fault tolerant messaging interface for fast and transparent recovery. In Proceedings of the 2014 IEEE 28th International Parallel and Distributed Processing Symposium, IPDPS '14, pages 1225-1234,

Washington, DC, USA, 2014. IEEE Computer Society.

[33] B. Schroeder, E. Pinheiro, and W.-D. Weber. Dram errors in the wild: A large-scale field study. In SIGMETRICS, 2009.

[34] M. Snir, R. W. Wisniewski, J. A. Abraham, S. V. Adve, S. Bagchi, P. Balaji, J. Belak, P. Bose, F. Cappello, B. Carlson, and et al. Addressing Failures in Exascale Computing. U.S. DoE, 2013.

[35] G. Sun. Exploring Memory Hierarchy Design with Emerging Memory Technologies. Lecture Notes in Electrical Engineering. Springer, 2013.

[36] K. Teranishi and M. A. Heroux. Toward Local Failure Local Recovery Resilience Model Using MPI-ULFM. In Proceedings of the 21st European MPI Users' Group Meeting, EuroMPI/ASIA '14, pages 51:51-51:56, New York, NY, USA, 2014. ACM.

[37] M. Turmon, R. Granat, D. Katz, and J. Lou. Tests and tolerances for high-performance software-implemented fault detection. IEEE Transactions on Computers, 52(5):579-591, 2003.

[38] G. Zheng, X. Ni, and L. V. Kalé. A scalable double in-memory checkpoint and restart scheme towards exascale. In IEEE/IFIP 42nd International Conference on Dependable Systems and Networks Workshops (DSN-W), pages 1-6, 2012. 